\title{
Author Correction: Increasing species sampling in chelicerate genomic-scale datasets provides support for monophyly of Acari and Arachnida
}

\author{
Jesus Lozano-Fernandez (10) 1,2,5,6, Alastair R. Tanner ${ }^{1,6}$, Mattia Giacomelli ${ }^{1}$, Robert Carton (1) 3 , Jakob Vinther (1) 1,2, \\ Gregory D. Edgecombe ${ }^{4} \&$ Davide Pisani (1) ${ }^{1,2}$
}

Correction to: Nature Communications https://doi.org/10.1038/s41467-019-10244-7, published online 24 May 2019.

The original version of this Article omitted the following from the Acknowledgements:

This research was funded by a NERC BETR grant (NE/P013678/1 to D.P. and J.V.), and the European Union's Horizon 2020 research and innovation programme under the Marie Skłodowska-Curie grant agreement (764840 to D.P. and M.G.).

This has now been corrected in both the PDF and HTML versions of the Article.

Published online: 01 October 2019

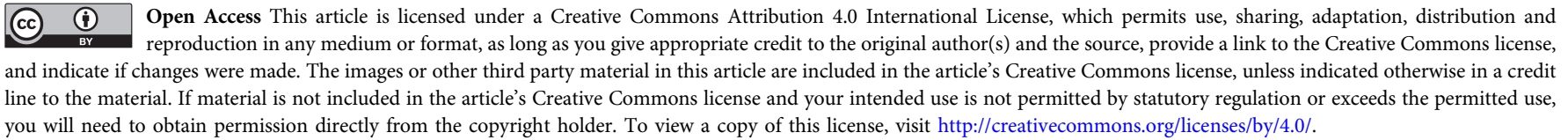

(C) The Author(s) 2019

\footnotetext{
${ }^{1}$ University of Bristol School of Biological Sciences, 24 Tyndall Avenue, Bristol BS8 1TQ, UK. ${ }^{2}$ University of Bristol School of Earth Sciences, 24 Tyndall Avenue, Bristol BS8 1TQ, UK. ${ }^{3}$ Department of Biology, The National University of Ireland Maynooth, Maynooth, Kildare, Ireland. ${ }^{4}$ Department of Earth Sciences, The Natural History Museum, Cromwell Road, London SW7 5BD, UK. ${ }^{5}$ Present address: Department of Evolutionary Biology, Ecology and Environmental Sciences, \& Biodiversity Research Institute (IRBio) Universitat de Barcelona, Avinguda Diagonal 643, Barcelona 08028, Spain. ${ }^{6}$ These authors contributed equally: Jesus Lozano-Fernandez, Alastair R. Tanner. Correspondence and requests for materials should be addressed to

G.D.E. (email: g.edgecombe@nhm.ac.uk) or to D.P. (email: davide.pisani@bristol.ac.uk)
} 